\title{
Probing the Debye dielectric relaxation in supercooled methanol
}

\author{
Xin Li, Zeming Chen, Yanqin Gao, Wenkang Tu and Li-Min Wang* \\ State Key Laboratory of Metastable Materials Science and Technology, College of Materials Science and Engineering, \\ Yanshan University, Qinhuangdao, Hebei, China
}

The explanation of the dielectric dynamics in methanol would offer knowledge of the Debye relaxation in supercooled monoalcohols. However, due to the fast crystallization, it is hard to attain the dynamics of pure methanol in the deeply supercooled region. In this paper, we studied the dynamics of methanol -2-ethyl-1-hexanol mixtures with methanol concentration up to 80 mol\% using dielectric and calorimetric measurements. Two main relaxations are detected in the dielectric spectra of the mixtures, and the slower one

OPEN ACCESS

Edited by:

Lothar Wondraczek,

University of Jena, Germany

Reviewed by:

Bruno Poletto Rodrigues, Otto-Schott-Institute of Materials

Research, Germany

Roger Jay Loucks,

Alfred University, USA

Kia L. Ngai,

National Research Council, Italy

*Correspondence:

Li-Min Wang,

State Key Laboratory of Metastable Materials Science and Technology,

College of Materials Science and Engineering, Yanshan University, 438 West Hebei Avenue, Qinhuangdao, Hebei 066004, China

limin_wang@ysu.edu.cn

Specialty section:

This article was submitted to Glass Science, a section of the journal Frontiers in Materials

Received: 01 March 2015 Accepted: 04 May 2015 Published: 03 June 2015

Citation:

Li X, Chen Z, Gao Y, Tu W and Wang L-M (2015) Probing the Debye dielectric relaxation in supercooled methanol.

Front. Mater. 2:41.

doi: 10.3389/fmats.2015.00041 generally reproduces the Debye relaxation features reported in earlier studies of other monoalcohols. The validity of the ideal mixing law is verified for the relaxation time of the slower dynamics in the mixtures. The results suggest that the Debye relaxation is present in the dielectric spectra of methanol. The temperature dependence of the Debye relaxation time is constructed for the supercooled methanol.

\section{Keywords: dielectric relaxation, glass transition, supercooled liquids, hydrogen-bond liquids, Debye relaxation, methanol}

\section{Introduction}

Common to glass-forming monoalcohols is the extra Debye type relaxations in dielectric spectra, manifested by the slower dynamics than the structural $(\alpha-)$ relaxation, which associates with liquid viscosity and calorimetric glass transitions (Hassion and Cole, 1955; Dannhauser, 1968; Kudlik et al., 1997; Petong et al., 1999; Johari et al., 2001; Wang and Richert, 2004; Fragiadakis et al., 2010; Pawlus et al., 2010; Gainaru et al., 2011; Power et al., 2011). A number of unique dynamic characters have been discovered for the Debye relaxation (Hansen et al., 1997; Wang and Richert, 2005a; Wang et al., 2005; Huth et al., 2007; Power et al., 2007; Jakobsen et al., 2008; Gainaru and Böhmer, 2010). The understanding of the Debye relaxation has been developed in the latest decades, and the chain-like associations imposed by intermolecular hydrogen-bond (H-bond) are considered to be responsible for the Debye relaxation, although the special relaxation might not be the direct signature of the overall reorientation of the associations as a whole (Fragiadakis et al., 2010; Gainaru et al., 2011; Singh and Richert, 2012). However, a complete understanding of the Debye relaxation is still unclear and, in particular, the formation of the associations (or clusters) corresponding to the Debye relaxation in H-bond liquids and the relation between the Debye and $\alpha$-relaxations have not yet been solved.

The studies of the dielectric dynamics in supercooled monoalcohols with small molecular size are expected to extend the structural knowledge (clusters) associated with the Debye relaxation. The exploration of the possibility that similar chain-like associations are present in methanol would consequently be of particular interest because of the smallest size among monoalcohols. Yet, the rapid crystallization in supercooled methanol near the glass transition region impedes the accurate determination of the dynamics and the glass transition temperature, $T_{\mathrm{g}}$ (Souda, 2004). Recent studies of the dielectric relaxation in supercooled ethanol found that it is not straightforward to confirm the 
Debye relaxation as the main relaxation becomes broader than pure Debye dynamics (Brand et al., 2002), especially when the plastic crystal is involved in ethanol.

In order to learn the dynamics of methanol, a common practice is to study the dynamics of binary mixtures of methanol (Sun et al., 2011). In the past few decades, aqueous solutions of methanol have been explored extensively; however, much less experimental studies are available for the dynamics in glass-forming mixtures composed of methanol and well-defined Debye liquids (Denney and Cole, 1955). Our recent studies of the mixtures composed of two monoalcohols suggest that the main relaxation in the dielectric spectra of the mixtures is still Debye typed, while the Debye relaxation disappears in monoalcohols diluted by non-Debye liquids at a specific mole fraction (Wang and Richert, 2005b; Wang et al., 2005). In this paper, we examine the dielectric behaviors in the glass-forming solutions of methanol mixed with 2-ethyl1-hexanol, a typical Debye liquid featured by the separated Debye and $\alpha$-relaxations and high glass-forming ability (Murthy, 1996; Wang and Richert, 2004; Wang et al., 2005; Jakobsen et al., 2008; Fragiadakis et al., 2010; Bauer et al., 2013). A broad composition range is explored up to $80 \mathrm{~mol} \%$ methanol, and the main dielectric relaxation with the slowest dynamics in the mixtures is focused. The dynamic features of the Debye relaxation in supercooled liquids are identified.

\section{Experimental}

Broadband dielectric relaxation measurements of the mixtures of methanol (Sigma Aldrich, 99\%) and 2-ethyl-1-hexanol (2E1H, Alfa Aesar, 99\%) are made in a Novocontrol broadband dielectric spectrometer (Concept 80 ) equipped with a liquid nitrogen cooling system (Chen et al., 2009). The dielectric relaxation is isothermally measured at each temperature by holding liquids between two separated brass electrodes by Teflon strips of thickness $25 \mu \mathrm{m}$. The scanning frequency range spans from $0.01 \mathrm{~Hz}$ to $10 \mathrm{MHz}$. The temperature is controlled by a Novocontrol Quatro controller with temperature accuracy within $0.1 \mathrm{~K}$. The dielectric data are analyzed to obtain the dynamic parameters in terms of Havriliak-Negami (HN) expressions (Havriliak and Negami, 1967),

$$
\varepsilon *(\omega)=\varepsilon_{\infty}+\sum_{\mathrm{i}} \frac{\Delta \varepsilon_{\mathrm{i}}}{\left(1+\left(i \omega \tau_{\mathrm{i}}\right)^{\alpha_{\mathrm{i}}}\right)^{\gamma_{\mathrm{i}}}}+\frac{\sigma_{\mathrm{dc}}}{i \varepsilon_{0} \omega}
$$

where $\varepsilon_{\infty}$ is the high-frequency dielectric constant, $\Delta \varepsilon_{\mathrm{i}}$ dielectric strength, $\tau_{\mathrm{i}}$ dielectric relaxation time, and $\alpha_{\mathrm{i}}$ and $\gamma_{\mathrm{i}}$ profile shape factors of the relaxation dispersion for the $i$ th dynamics. With $\alpha=1$, the HN equation becomes the Cole-Davidson (CD) equation, while $\gamma=1$ leads to the Cole-Cole (CC) equation. $\sigma_{\mathrm{dc}}$ is the dc conductivity.

The calorimetric glass transition of the mixtures is recorded in a Perkin-Elmer (PE) Diamond differential scanning calorimetry (DSC) at fixed cooling and heating rates of $20 \mathrm{~K} / \mathrm{min}$. The instrument is calibrated by indium and cyclohexane for the high and low temperature, following a previous procedure described elsewhere (Wang et al., 2002). The glass transition temperatures, $T_{\text {g-cal, }}$ are determined by the onset temperatures of the heat capacity jumps from glasses to supercooled liquids.

\section{Results}

The dielectric loss spectra measured for the methanol $-2 \mathrm{E} 1 \mathrm{H}$ mixtures are shown in Figure 1. In Figures 1A,B, two relaxations I and II are easily seen in the isothermal spectra of the mixtures with 10 and $30 \mathrm{~mol} \%$ methanol (i.e., $10 \mathrm{M}-90 \mathrm{H}$ and $30 \mathrm{M}-70 \mathrm{H}$ ), in analogy to the dielectric behaviors of pure $2 \mathrm{E} 1 \mathrm{H}$ (Wang and Richert, 2004). The slow relaxation is consequently expected to be Debye typed. Fits to the loss peaks of the $10 \mathrm{M}-90 \mathrm{H}$ mixtures show a CD type profile for the Debye relaxation with $\gamma_{\mathrm{I}}=1$, while the $\alpha$-relaxation shows typical $\mathrm{HN}$ profile, giving typical values of $\alpha_{\text {II }} \sim 0.75$ and $\gamma_{\text {II }} \sim 0.55$. As the concentration of methanol increases, the CC type profiles for the slow relaxation are favored based on the comparison of the fitting quality. In Figure 1B, a fitting with $\alpha_{\mathrm{I}}=0.9$ and $\gamma_{\mathrm{I}}=1$ for the $163 \mathrm{~K}$ curve of the $30 \mathrm{M}-70 \mathrm{H}$ mixture well explains the main relaxation, while the shape parameters of the $\alpha$-relaxation are comparable with those in the $10 \mathrm{M}-90 \mathrm{H}$ mixture. The dielectric spectra obtained in the mixtures with 60 mol\% methanol are presented in Figure 1C. It is difficult to distinguish two dynamics, and apparently one HN equation seems to basically explain the loss spectra. Figure 1D shows the dielectric spectra of the mixtures with $80 \mathrm{~mol} \%$ methanol, and two relaxations are resolved and the $\mathrm{CC}$ equation is also proven to be suitable for the slow relaxation.

Figure 2 presents a close inspection of the relaxation dispersion profiles of the mixtures with the loss spectra normalized by the loss maxima positioned at $1-10 \mathrm{~Hz}$. It is seen that as the fraction of methanol increases, the slope of the low-frequency flank of the slow relaxation reduces continuously from unity. Further analyses of the relaxation dispersion parameters (see Figure 4) suggest that the continuous broadening of the relaxation I cannot be explained by the interference of the relaxation II, although the dynamic separation is reduced between the two relaxations.

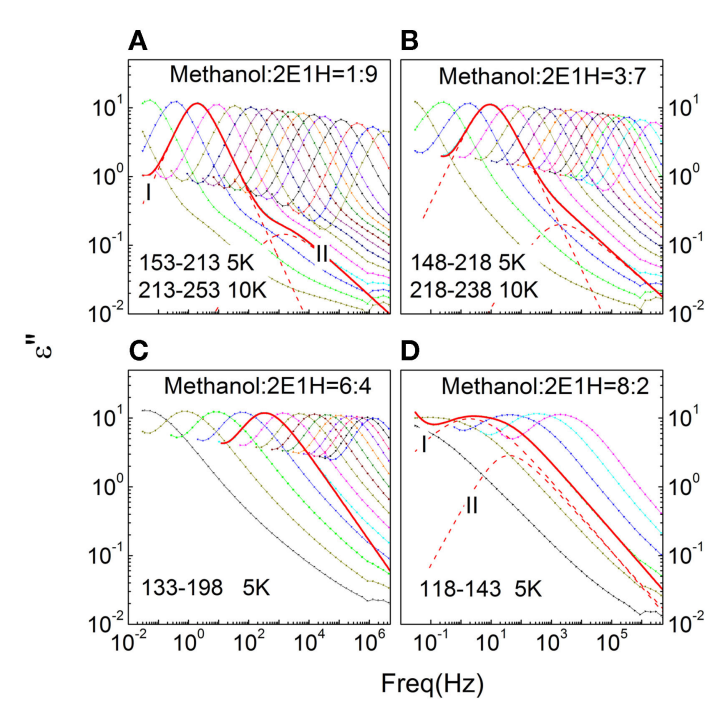

FIGURE 1 | Frequency-dependent dielectric loss $\left(\varepsilon^{\prime \prime}\right)$ spectra of methanol - 2-ethyl-1-haxanol (2E1H) mixtures with four mole fractions (A-D). The fits of Havriliak-Negami equation to the representative curves are shown. 


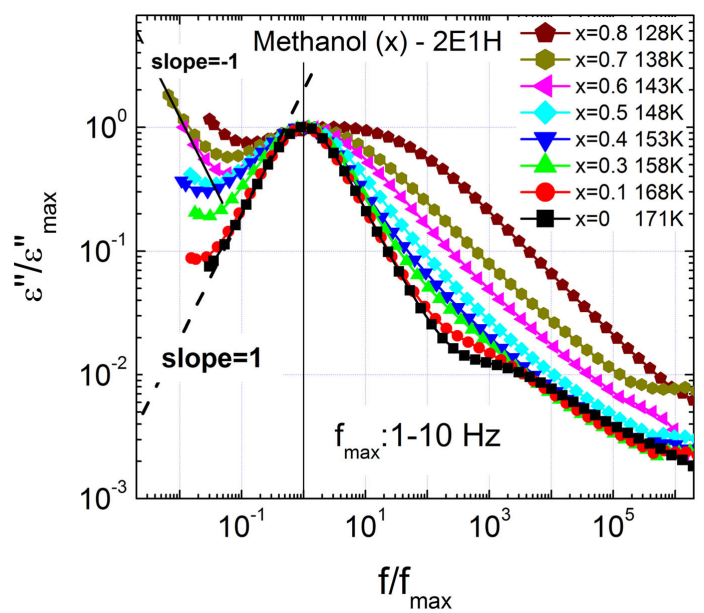

FIGURE 2 | Normalized dielectric loss spectra of methanol - 2-ethyl1-haxanol $(2 \mathrm{E} 1 \mathrm{H})$ mixtures, showing increasing broadening of the slow relaxation. The data fitting show Cole-Cole equation explains the slow relaxation with higher fitting quality.

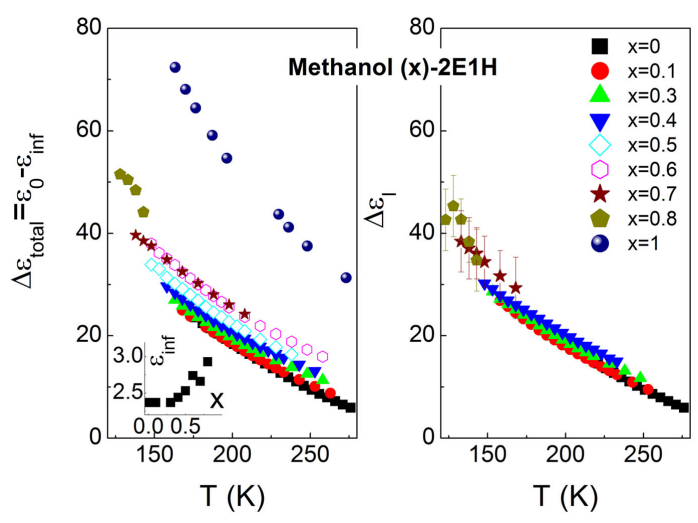

FIGURE 3 | Total dielectric relaxation strength (left panel) and relaxation strength of the relaxation I (right panel) . The high-frequency dielectric constant is shown in the inset.

The relaxation strength and relaxation dispersion of the dynamics I and II are presented in Figures 3 and 4. It is seen that in the range of the methanol mole fraction below 0.7 , the total relaxation strength slightly increases, while for the higher fraction, the large enhancement in the relaxation strength is observed, in particular when the dielectric data of pure methanol (Denney and Cole, 1955 ) is added. The similar case is also observed for the relaxation strength of the relaxation I. The numerical comparison finds that the relaxation I basically explains the total relaxation strength for all the mixtures, suggesting that the relaxation II is relatively weak. A close inspection of the relaxation II also finds a continuous increase of the relaxation strength with increased concentration of methanol. Figure 4 shows the relaxation dispersion parameters of the two relaxations, and the CC type character is revealed for the relaxation I of the mixtures as the methanol mole fraction exceeds $0.3 . \alpha_{\mathrm{I}}$ is observed to continuously reduce, indicative of

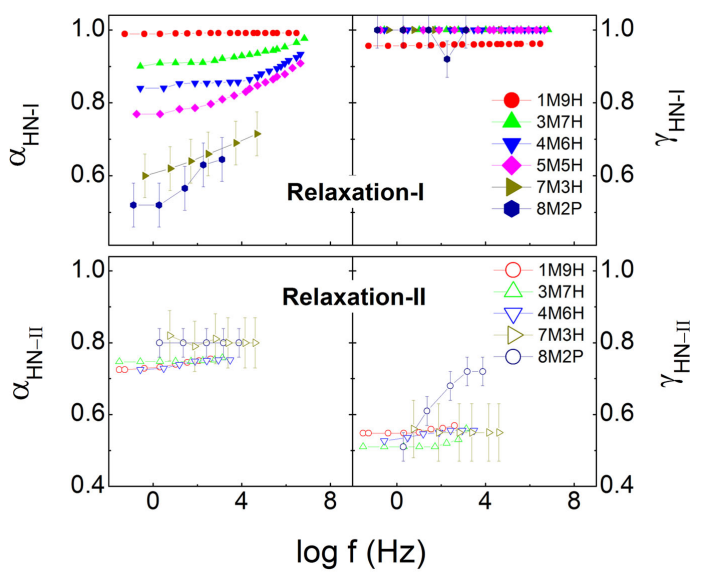

FIGURE 4 | Relaxation dispersion parameters, $\alpha_{\mathrm{HN}}$ and $\gamma_{\mathrm{HN}}$ in Havriliak-Negami equation for relaxations I and II.
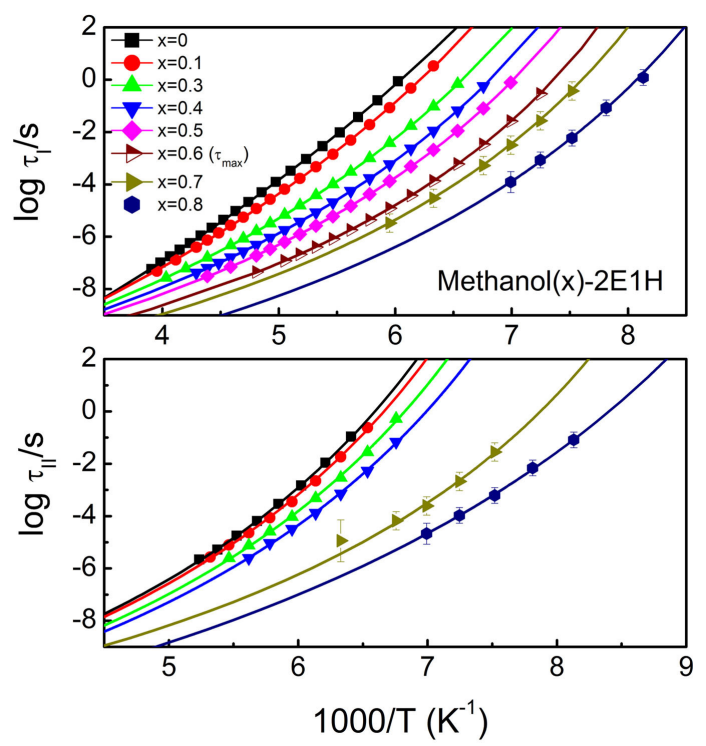

FIGURE 5 | Activation plots for the relaxations I (upper panel) and II (lower panel) of methanol - 2-ethyl-1-haxanol (2E1H) mixtures. The relaxation time is obtained from Havriliak-Negami equation except for the mixture with $60 \mathrm{~mol} \%$ methanol where the time corresponding to the loss maximum is used since two relaxations can not be separated. The fits of the Vogel-Fulcher-Tammann equation to the relaxation times of the two relaxations are presented by the lines. $x$ is the mole fraction of methanol.

increased broadening of the relaxation I. In contrast, the relaxation dispersion parameters, $\alpha_{\text {II }}$ and $\gamma_{\text {II }}$, of the relaxation II basically keep constant, consistent with the temperature dependence of the $\alpha$-relaxation dispersion in generic liquids, where the relaxation width either keeps constant or decreases in the supercooled regions as temperature increases (Dixon et al., 1990; Olsen et al., 2001; Wang and Richert, 2007).

Figure 5 presents the reciprocal temperature dependence of the relaxation times for the relaxations I and II of the mixtures. The Vogel-Fulcher-Tammann (VFT) equation, $\log _{10} \tau=A+B /\left(T-T_{0}\right)$, where $\tau$ is the relaxation time and $A, B$, and $T_{0}$ are constants, is 
used to analyze the data. The kinetic glass transition temperature, $T_{\mathrm{g} \text {-kin }}$ is usually defined by a temperature where the relaxation time approaches $100 \mathrm{~s}$ (Angell et al., 2000). And thus, two $T_{\mathrm{g} \text {-kin }}$ values can be obtained for the methanol-deficit mixtures, denoted by $T_{\tau_{\mathrm{D}}=100 \mathrm{~s}}$ for the relaxation I and $T_{\tau_{\alpha}=100 \mathrm{~s}}$ for the relaxation II. $T_{\mathrm{g}}$ can also be determined from calorimetric measurements, and Figure 6 presents the calorimetric results of the mixtures with $T_{\text {g-cal }}$ defined by the onset transition temperature in the heatflow curves from glassy to supercooled liquid states. Only one glass transition is clearly identified, and this suggests that no phase separation occurs in the mixtures.

\section{Discussion}

Two relaxations are observed in the dielectric spectra of the methanol-2E1H mixtures at the methanol-rich and 2E1H-rich compositions. For the $2 \mathrm{E} 1 \mathrm{H}$-rich compositions, it can be easily concluded that the relaxation I is of the same nature as the Debye relaxation detected in pure $2 \mathrm{E} 1 \mathrm{H}$, since the Debye relaxation has been proven not to disappear in the diluted monoalcohols until a certain concentration (Wang et al., 2005; El Goresy and Böhmer, 2008). In contrast, the identification of the slow relaxation (I) in the methanol-rich compositions might not be straightforward. Fortunately, the unique dynamic features in supercooled states found in earlier publications can be used to serve as the basic criteria to distinguish the Debye relaxation from $\alpha$-relaxation in pure liquids or liquid mixtures (Wang and Richert, 2005a). Naturally, two prominent dynamic features of the Debye relaxation will be examined for the methanol-rich mixtures, one being the difference between the kinetic $T_{\mathrm{g}}$ in terms of the Debye relaxation time and the calorimetrically measured $T_{\mathrm{g}}$ (Wang and Richert, 2005a; Wang et al., 2008) and the other the ideal mixing law of

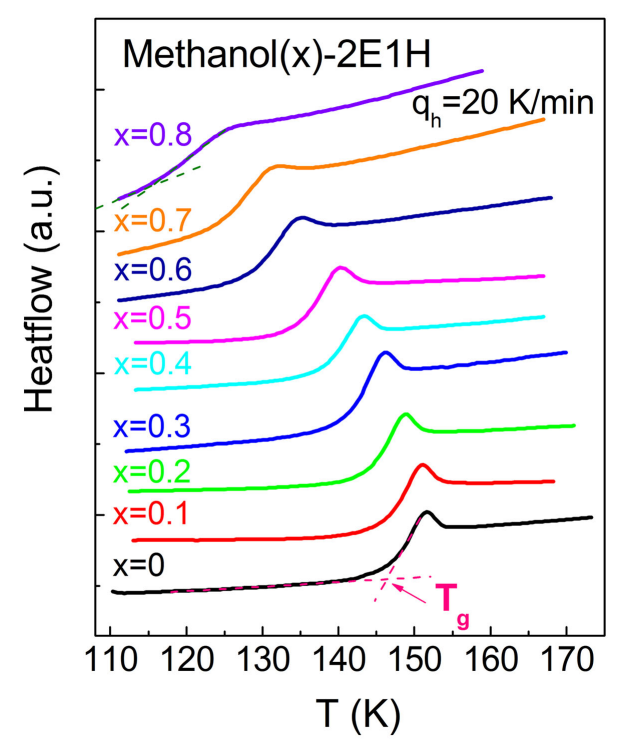

FIGURE 6 | Glass transition heat capacity curves measured at the heating rate of $20 \mathrm{~K} / \mathrm{min}$ for the methanol - 2-ethyl-1-haxanol (2E1H) mixtures quenched at the same cooling rate. The calorimetric glass transition temperature, $T_{g-\text { cal }}$, is defined. $x$ is the mole fraction of methanol. the Debye relaxation time in the binary mixtures of two primary monoalcohols (Wang and Richert, 2005b).

\section{Calorimetric and Kinetic $\boldsymbol{T}_{\mathrm{g}}$}

For generic (non-Debye) molecular liquids, $T_{\mathrm{g} \text {-kin }}$ derived from the dielectric $\alpha$-relaxation is found to relate closely to the calorimetric $T_{\text {g-cal, }}$ which is defined by a thermal procedure with fixed cooling and subsequently heating rates, typically $20 \mathrm{~K} / \mathrm{min}$, and, generally, $T_{\text {g-kin }}$ of generic liquids is constantly $0-3 \mathrm{~K}$ lower than $T_{\mathrm{g} \text {-cal }}$ (Wang et al., 2008; Li et al., 2014). Instead, for Debye liquids, if the Debye relaxation time $\tau_{\mathrm{D}}$ is used to define $T_{\mathrm{g} \text {-kin }}, T_{\mathrm{g} \text {-kin }}$ would exceed $T_{\text {g-cal }}$, and in some cases, the difference can be as large as $7 \mathrm{~K}$ (Wang et al., 2008). The calorimetric and kinetic $T_{\mathrm{g}}$ values of the methanol - 2E1H mixtures are compared in Figure 7, where $T_{\text {g-kin }}$ are calculated by the relaxations I and II, respectively. It is seen that $T_{\tau_{\mathrm{D}}=100 \mathrm{~s}}$ is fundamentally greater than $T_{\mathrm{g} \text {-cal }}$, while $T_{\tau_{\alpha}=100 \mathrm{~s}}$ is smaller than $T_{\text {g-cal }}$ in the whole composition range, similar to the relation between the kinetic and calorimetric $T_{\mathrm{g}}$ 's of Debye liquids. It is observed that the difference between $T_{\tau_{\mathrm{D}}=100 \mathrm{~s}}$ and $T_{\tau_{\alpha}=100 \mathrm{~s}}$ is relatively large at both methanol-rich and methanol-deficit compositions for the methanol-2E1H mixtures, and a minimum is expected at an intermediate composition of 50-60 mol\%. The inset of Figure 7 presents the $T_{\mathrm{g}}$ results of the mixtures of two primary monoalcohols, 2E1H and 2-methyl-1-butanol (2M1B) (Wang and Richert, 2005b), revealing the similar minimum of $T_{\tau_{\mathrm{D}}=100 \mathrm{~s}}$ and $T_{\tau_{\alpha}=100 \mathrm{~s}}$ difference at a composition range of $30-50 \mathrm{~mol} \%$ of $2 \mathrm{M} 1 \mathrm{~B}$. When comparing the two mixing systems of methanol vs. $2 \mathrm{E} 1 \mathrm{H}$ and $2 \mathrm{E} 1 \mathrm{H}$ vs. $2 \mathrm{M} 1 \mathrm{~B}$, the difference is obvious that the broadening of the relaxation I in the dielectric spectra of the former system is much more pronounced, in particular at methanol-rich compositions and, in contrast, only tiny broadening is shown in the latter

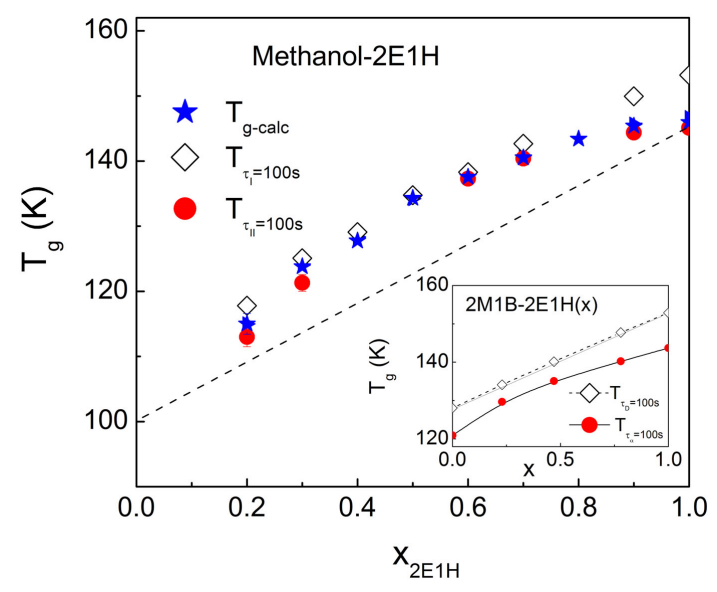

FIGURE 7 | Comparison of the glass transition temperatures defined in calorimetric and kinetic measurements for the methanol - $2 \mathrm{E} 1 \mathrm{H}$ mixtures. Calorimetric $T_{\text {g-cal }}$ is defined as the onset temperature in the heat capacity jump from the glassy to liquid states as shown in Figure 4, while kinetic $T_{\tau_{\mathrm{D}}=100 \mathrm{~s}}$ of the relaxation I and $T_{\tau_{\alpha}=100 \mathrm{~s}}$ of the relaxation II are determined by a temperature at which the dielectric relaxation time is $100 \mathrm{~s}$. $T_{\tau_{\mathrm{D}}=100 \mathrm{~s}}$ and $T_{\tau_{\alpha}=100 \mathrm{~s}}$ in the mixtures composed of $2 \mathrm{E} 1 \mathrm{H}$ and 2-methyl-1butanol (2M1B) from Wang and Richert (2005b) are plotted in the inset. $x$ is the mole fraction of $2 \mathrm{E} 1 \mathrm{H}$. 
system occurring at the intermediate compositions. The larger broadening in the methanol-2E1H mixtures might be attributed to the large size difference between the two constituents. The composition dependence of calorimetrical $T_{\mathrm{g}}$ of the mixtures allows to evaluate roughly $T_{\mathrm{g}}$ of methanol, yielding a value of $\sim 100 \mathrm{~K}$, which is comparable with the reported value of $103 \mathrm{~K}$ of a hyperquenched glass (Sugisaki et al., 1968).

\section{Ideal Mixing Law of the Debye Relaxation Time}

Recent dielectric relaxation studies of mixtures composed of two primary monoalcohols found that the Debye relaxation time follows the ideal mixing law, i.e., $\log \tau_{\mathrm{x}}=x \log \tau_{\mathrm{D} 1}+(1-x) \log \tau_{\mathrm{D} 2}$, where $\tau_{\mathrm{x}}$ is the Debye relaxation of the mixture at a mole fraction of $x, \tau_{\mathrm{Di}}$ is the Debye relaxation time of the $i$ th primary monoalcohol (Wang and Richert, 2005b). In order to clarify whether the ideal mixing rule applies to the methanol-2E1H mixtures in the deeply supercooled regimes, a reliable practice is to compare the calculated Debye relaxation times of pure methanol at a temperature yielded in terms of the ideal mixing rule of the data of pure $2 \mathrm{E} 1 \mathrm{H}$ and a specified mixture, and if the Debye relaxation is not present in the dielectric spectra of pure methanol, the calculated values would not match well. Figure 8 reproduces the experimental data in upper panel of Figure 5, and the validity of the ideal mixing law is tested at a few representative temperatures at which the reliable experimental data of $2 \mathrm{E} 1 \mathrm{H}$ and the mixtures are achieved. Interestingly, the calculated Debye relaxation times of pure methanol at each temperature are found to be comparable with small fluctuation, indicated by the error bars. The averaged times are denoted by the purple pentagons in Figure 8.

Based on the calculated Debye relaxation times of methanol, the temperature dependence of the Debye relaxation time is expressed by a VFT equation of $\log \tau_{\mathrm{D}}=12.4+459.4 /(T-74.1)$, as shown

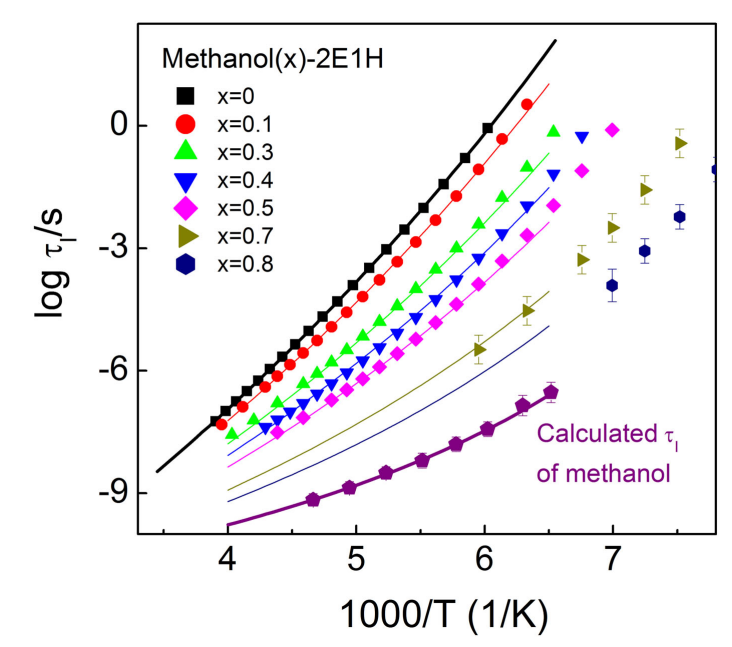

FIGURE 8 | Temperature dependence of the relaxation time of the relaxation I for the methanol - 2-ethyl-1-haxanol (2E1H) mixtures. Purple pentagon denotes the average Debye relaxation time of pure methanol, which is calculated when the ideal mixing law is verified. The thin lines are the calculated relaxation time for the mixtures in terms of the ideal mixing law based on the data of pure methanol and $2 \mathrm{E} 1 \mathrm{H}$ (thick lines). by the thick purple line in Figure 8. The fit of the VFT equation to the temperature dependence of the Debye relaxation time for $2 \mathrm{E} 1 \mathrm{H}$ gives $\log \tau_{\mathrm{D}}=15.9+1738.7 /(T-56.1)$. Using the two VFT equations of methanol and $2 \mathrm{E} 1 \mathrm{H}$, the Debye relaxation time of each mixture is yielded, as denoted by the thin lines. It is seen that the calculated lines basically agree with the experimental data for each mixture. The validity of the ideal mixing law is consequently verified for the dielectric relaxation of the methanol-2E1H mixtures, suggesting the dynamics in the methanol-2E1H mixtures shares the same feature of the ideal mixing law as observed in earlier study of the mixtures of two primary monoalcohols. The results offer new evidence for the presence of the Debye relaxation in the dielectric spectra of pure methanol. Two main relaxations have been reported in the dielectric relaxations of ethanol (Brand et al., 2000) and propanol (Hansen et al., 1997), and it appears that the slow relaxations are of the Debye relaxation character. Further, it is inferred that similar intermolecular structures (clusters) form in the monoalcohols, which are responsible for the Debye relaxation.

The activation plot of the Debye relaxation time of methanol is presented in Figure 9, covering the normal and supercooled liquid regimes. The data reported in earlier studies derived from the high-frequency measurements are also given in Figure 9. One can see that the Debye relaxation times calculated in the present study are consistent with most of the earlier measurements (Jordan et al., 1978; Bertolini et al., 1983; Barthel et al., 1990), except for the data reported by Denney and Cole (1955), which shows an obvious discrepancy from others. It should be noticed that the relaxation time in the study by Denney and Cole (1955) was determined using two or three additive Debye relaxations to analyze the CC ( $\varepsilon^{\prime}$ vs. $\varepsilon^{\prime \prime}$ ) plots of the dielectric spectra, while the data reported in the latter publications are obtained from the frequent-dependent isothermal dielectric spectra measurements. The fits to the Debye relaxation

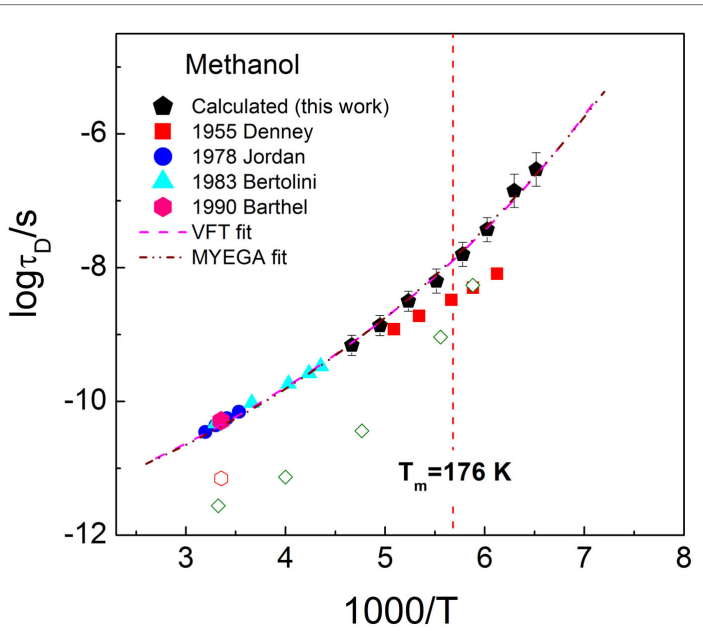

FIGURE 9 | Activation plot of methanol in temperature range covering normal and supercooled liquids. The data from earlier studies are also shown. The lines are guides for the eye, represented by the Vogel-FulcherTammann equation of $\log \tau_{\mathrm{D}}=-12.4+459.4 /(T-74.1)$ (pink dash line) and Mauro-Yue-Ellison-Gupta-Allan equation of $\log \tau_{\mathrm{D}}=-12.2+335.3 / T \times$ $\exp (144.5 / T)$ (green short dash line). The faster $\alpha$-relaxation times are shown from Barthel et al. (1990) (experimental, open hexagon) and Ngai and Roland (1997) (MD, open diamond). 
times from our work and reported by Jordan et al. (1978), Bertolini et al. (1983), and Barthel et al. (1990) are attempted in Figure 9 using the VFT equation and Mauro-Yue-Ellison-Gupta-Allan equation of $\log \tau_{\mathrm{D}}=A+K / T \times \exp (C / T)$ (Mauro et al., 2009), where $A, K$, and $C$ are constants, showing excellent consistency.

The $\alpha$-relaxation time of methanol at temperature around the melting point was analyzed using dynamic simulation by Ngai and Roland (1997). The data are represented in Figure 9 by the open diamond, and the data are comparable with the time of the faster relaxation determined experimentally in the study by Barthel et al. (1990), as denoted by the open hexagon. The increased separation between the Debye and $\alpha$-relaxation times with temperature is visible. When comparing the $\mathrm{MD} \alpha$-relaxation time with the Debye relaxation time reported in the study by Denney and Cole (1955), it appears that the latter data might be subjected to considerable error.

\section{Kirkwood Factors}

The Kirkwood factor is a crucial quantity to obtain the knowledge of liquid structures, which can be calculated in terms of the Kirkwood-Fröhlich equation (Kirkwood, 1939).

$$
\frac{\left(\varepsilon_{\mathrm{s}}-\varepsilon_{\infty}\right)\left(2 \varepsilon_{\mathrm{s}}+\varepsilon_{\infty}\right)}{\varepsilon_{\mathrm{s}}\left(\varepsilon_{\infty}+2\right)^{2}}=\frac{N_{\mathrm{A}} \rho \mu_{0}{ }^{2}}{9 \varepsilon_{0} M k_{\mathrm{B}} T} g_{\mathrm{K}}=y g_{\mathrm{K}}
$$

where $\varepsilon_{s}$ is the static permittivity, $\varepsilon_{\infty}$ is the permittivity in the limit of high frequencies as defined in eq. (1). $N_{\mathrm{A}}$ is Avogadro's number, $\rho$ the density of the liquid at a temperature $T, \mu_{0}$ the dipole moment of the isolated molecule, $\varepsilon_{0}$ the absolute permittivity of vacuum, $M$ the molecular weight, and $k_{\mathrm{B}}$ Boltzmann's constant. Experimentally determined $g_{\mathrm{K}}$ can explain additional dipole correlations generated by interactions such as H-bond in alcohols (Singh and Richert, 2012; Li et al., 2014). $g_{\mathrm{K}}=1$ means the absence of any additional correlation, and $g_{\mathrm{K}}>1$ denotes an enhancement of the effective dipole moment induced by the parallel alignment, while $g_{\mathrm{K}}<1$ reflects antiparallel alignment due to dipole cancelation effects (Wang and Richert, 2005a). For binary mixtures, a rough approximation for the average dipole moment $\mu_{0}$ can be referred to (Lu et al., 2009),

$$
\mu_{0}=\left(v_{1} \mu_{1}^{2}+v_{2} \mu_{2}^{2}\right)^{1 / 2}
$$

$\nu_{\mathrm{i}}$ is the volume fraction of component $i$.

The dipole moments of methanol and $2 \mathrm{E} 1 \mathrm{H}$ were reported to be 1.69 D (Caldwell and Kollman, 1995) and 1.74 D (McClellan,

\section{References}

Angell, C. A., Ngai, K. L., McKenna, G. B., McMillan, P. F., and Martin, S. W. (2000). Relaxation in glassforming liquids and amorphous solids. J. Appl. Phys. 88, 3113-3157. doi:10.1063/1.1286035

Barthel, J., Bachhuber, K., Buchner, R., and Hetzenauer, H. (1990). Dielectric spectra of some common solvents in the microwave region water and lower alcohols. Chem. Phys. Lett. 165, 369-373. doi:10.1016/0009-2614(90)87204-5

Bauer, S., Wittkamp, H., Schildmann, S., Frey, M., Hiller, W., Hecksher, T., et al. (2013). Broadband dynamics in neat 4-methyl-3-heptanol and in mixtures with 2-ethyl-1-hexanol. J. Chem. Phys. 139, 134503. doi:10.1063/1.4821229

Bertolini, D., Cassettari, M., and Salvetti, G. (1983). The dielectric properties of alcohols-water solutions. I. The alcohol rich region. J. Chem. Phys. 78, 365-372. doi: $10.1063 / 1.444510$

Brand, R., Lunkenheimer, P., and Loidl, A. (2002). Relaxation dynamics in plastic crystals. J. Chem. Phys. 116, 10386-10401. doi:10.1063/1.1477186
1963), and the temperature dependences of the densities are available in literatures for the two monoalcohols. (Nikam et al., 1998; Zorębski et al., 2011) Three mixtures are chosen to calculate the $g_{\mathrm{K}}$ factors at temperatures near the glass transitions using the dielectric relaxation strength shown in Figure 3. The $g_{\mathrm{K}}$ factors of $4.56,4.06$, and 4.05 are yielded for the $40 \mathrm{M}-60 \mathrm{H}(158 \mathrm{~K})$, $60 \mathrm{M}-40 \mathrm{H}(158 \mathrm{~K})$, and $80 \mathrm{M}-20 \mathrm{H}(143 \mathrm{~K})$ mixtures, respectively, similar to the high values reported for the Debye relaxations in Debye liquids (Wang and Richert, 2005a). According to the explanation of the Debye relaxations (Fragiadakis et al., 2010; Gainaru et al., 2010, 2011; Singh and Richert, 2012; Pawlus et al., 2013), the high $g_{\mathrm{K}}$ factors suggest the formation of the chain-like intermolecular associations in the mixtures driven by the $\mathrm{H}$-bond among methanol and $2 \mathrm{E} 1 \mathrm{H}$ molecules. The structural studies of liquid and solid methanol indeed argued the formation of the intermolecular H-bonded chains (Sindzingre and Klein, 1992; Guo et al., 2003).

\section{Conclusion}

The dynamic analyses together with the calculation of the Kirkwood factors of the methanol-2E1H mixtures suggest that the Debye relaxation is present in the dielectric spectra of pure methanol. The conclusion is also supported by a recent study of the comparison of the dielectric and low-frequency Raman spectra of methanol, finding that the slow dielectric dynamics, which is the Debye relaxation, does not correspond to the Raman spectrum, and the faster processes reflect common microscopic dynamics (Fukasawa et al., 2005). The validity of the ideal mixing law for the Debye relaxation time of the mixtures advances the construction of the temperature dependence of the Debye relaxation time of the pure methanol, which will facilitate the evaluation of the dynamics in deeply supercooled methanol. In addition, broadened Debye relaxation is expected in the dielectric spectra of the methanol, and this is quite different from earlier observation of the Debye liquids.

\section{Acknowledgments}

This work was supported by National Basic Research Program of China (973 Program No. 2015CB856805), NSFC (Grant No. 51131002/51421091/51271160/11474247), and Natural Science Foundation of Hebei Province (No. A2014203260).

Brand, R., Lunkenheimer, P., Schneider, U., and Loidl, A. (2000). Excess wing in the dielectric loss of glass-forming ethanol: A relaxation process. Phys. Rev. E 62, 8878-8883. doi:10.1103/PhysRevB.62.8878

Caldwell, J. W., and Kollman, P. A. (1995). Structure and properties of neat liquids using nonadditive molecular dynamics: Water, methanol, and $\mathrm{N}$-methylacetamide. J. Phys. Chem. 99, 6208-6219. doi:10.1021/j100016a067

Chen, Z. M., Zhao, Y., and Wang, L.-M. (2009). Enthalpy and dielectric relaxations in supercooled methyl m-toluate. J. Chem. Phys. 130, 204515. doi:10.1063/1.3142142

Dannhauser, W. (1968). Dielectric relaxation in isomeric octyl alcohols. J. Chem. Phys. 48, 1918-1923. doi:10.1063/1.1668990

Denney, D. J., and Cole, R. H. (1955). Dielectric properties of methanol and methanol-1-propanol solutions. J. Chem. Phys. 23, 1767-1772. doi:10.1063/1.1740577

Dixon, P. K., Wu, L., Nagel, S. R., Williams, B. D., and Carini, J. P. (1990). Scaling in the relaxation of supercooled liquids. Phys. Rev. Lett. 65, 1108-1111. doi:10.1103/ PhysRevLett.65.1108 
El Goresy, T., and Böhmer, R. (2008). Diluting the hydrogen bonds in viscous solutions of n-butanol with n-bromobutane: A dielectric study. J. Chem. Phys. 128, 154520. doi:10.1063/1.2903403

Fragiadakis, D., Roland, C. M., and Casalini, R. (2010). Insights on the origin of the Debye process in monoalcohols from dielectric spectroscopy under extreme pressure conditions. J. Chem. Phys. 132, 144505. doi:10.1063/1.3374820

Fukasawa, T., Sato, T., Watanabe, J., Hama, Y., Kunz, W., and Buchner, R. (2005). Relation between dielectric and low-frequency Raman spectra of hydrogen-bond liquids. Phys. Rev. Lett. 95, 197802. doi:10.1103/PhysRevLett.95.197802

Gainaru, C., and Böhmer, R. (2010). Coupling of the electrical conductivity to the structural relaxation, absence of physical aging on the time scale of the Debye process, and number of correlated molecules in the supercooled monohydroxy alcohol 2-ethylhexanol. J. Non Cryst. Solids 356, 542-546. doi:10.1016/j. jnoncrysol.2009.07.030

Gainaru, C., Kastner, S., Mayr, F., Lunkenheimer, P., Schildmann, S., Weber, H. J., et al. (2011). Hydrogen-bond equilibria and lifetimes in a monohydroxy alcohol. Phys. Rev. Lett. 107, 118304. doi:10.1103/PhysRevLett.107.118304

Gainaru, C., Meier, R., Schildmann, S., Lederle, C., Hiller, W., Rössler, E. A., et al. (2010). Nuclear-magnetic-resonance measurements reveal the origin of the Debye process in monohydroxy alcohols. Phys. Rev. Lett. 105, 258303. doi:10.1103/ PhysRevLett.105.258303

Guo, J.-H., Luo, Y., Augustsson, A., Kashtanov, S., Rubensson, J.-E., Shuh, D. K., et al. (2003). Molecular structure of alcohol-water mixtures. Phys. Rev. Lett. 91, 157401. doi:10.1103/PhysRevLett.91.157401

Hansen, C., Stickel, F., Berger, F. T., Richert, R., and Fischer, E. W. (1997). Dynamics of glass-forming liquids. III. Comparing the dielectric $\alpha$ - and $\beta$-relaxation of 1-propanol and o-terphenyl. J. Chem. Phys. 107, 1086-1092. doi:10.1063/1.474456

Hassion, F. X., and Cole, R. H. (1955). Dielectric properties of liquid ethanol and 2-propanol. J. Chem. Phys. 23, 1756-1761. doi:10.1063/1.1740575

Havriliak, S., and Negami, S. (1967). A complex plane representation of dielectric and mechanical relaxation processes in some polymers. Polymer 8, 161-210. doi:10.1016/0032-3861(67)90021-3

Huth, H., Wang, L.-M., Schick, C., and Richert, R. (2007). Comparing calorimetric and dielectric polarization modes in viscous 2-ethyl-1-hexanol. J. Chem. Phys. 126, 104503. doi:10.1063/1.2539105

Jakobsen, B., Maggi, C., Christensen, T., and Dyre, J. C. (2008). Investigation of the shear-mechanical and dielectric relaxation processes in two monoalcohols close to the glass transition. J. Chem. Phys. 129, 184502. doi:10.1063/1.3007988

Johari, G. P., Kalinovskaya, O. E., and Vij, J. K. (2001). Effects of induced steric hindrance on the dielectric behavior and $\mathrm{H}$ bonding in the supercooled liquid and vitreous alcohol. J. Chem. Phys. 114, 4634-4642. doi:10.1063/1.1346635

Jordan, B. P., Sheppard, R. J., and Szwarnowski, S. (1978). The dielectric properties of formamide, ethanediol and methanol. J. Phys. D Appl. Phys. 11, 695-701. doi:10.1088/0022-3727/11/5/013

Kirkwood, J. G. (1939). The dielectric polarization of polar liquids. J. Chem. Phys. 7, 911-919. doi:10.1063/1.1750343

Kudlik, A., Tschirwitz, C., Benkhof, S., Blochowicz, T., and Rössler, E. (1997). Slow secondary relaxation process in supercooled liquids. Europhys. Lett. 40, 649-654. doi:10.1209/epl/i1997-00518-y

Li, X., Chen, Z. M., Li, Z. J., Gao, Y. Q., Tu, W. K., Li, X. Q., et al. (2014). Comparative study of dynamics in glass forming mixtures of Debye-type $N$-ethylacetamide with water, alcohol, and amine. J. Chem. Phys. 141, 104506. doi:10.1063/1.4895066

Lu, Z. J., Manias, E., Macdonald, D. D., and Lanagan, M. (2009). Dielectric relaxation in dimethyl sulfoxide/water mixtures studied by microwave dielectric relaxation spectroscopy. J. Phys. Chem. A 113, 12207-12214. doi:10.1021/jp9059246

Mauro, J. C., Yue, Y. Z., Ellison, A. J., Gupta, P. K., and Allan, D. C. (2009). Viscosity of glass-forming liquids. Proc. Natl. Acad. Sci. U.S.A. 24, 19780-19784. doi:10.1073/ pnas.0911705106

McClellan, A. L. (1963). Tables of Experimental Dipole Moments, Vol. 3. San Francisco, CA: W.H. Freeman and Co.

Murthy, S. S. N. (1996). Dielectric relaxation in monohydroxy alcohols and its connection to the glass transition process. J. Phys. Chem. 100, 8508-8517. doi:10.1021/ jp953596z

Ngai, K. L., and Roland, C. M. (1997). Short-time relaxational dynamics of the "strong" glass-former methanol. J. Phys. Chem. B 101, 4437-4441. doi:10.1021/jp963352u

Nikam, P. S., Shirsat, L. N., and Hasan, M. (1998). Density and viscosity studies of binary mixtures of acetonitrile with methanol, ethanol, propan-1-ol, propan-2-ol, butan-1-ol, 2-methylpropan-1-ol, and 2-methylpropan-2-ol at (298.15, 303.15, 308.15, and 313.15) K. J. Chem. Eng. Data 43, 732-737. doi:10.1021/je980028e
Olsen, N. B., Cristensec, T., and Dyre, J. C. (2001). Time-temperature superposition in viscous liquids Phys. Rev. Lett. 86, 1271-1274. doi:10.1103/PhysRevLett.86.1271

Pawlus, S., Klotz, S., and Paluch, M. (2013). Effect of compression on the relationship between viscosity and dielectric relaxation time in hydrogen-bonded primary alcohols. Phys. Rev. Lett. 110, 173004. doi:10.1103/PhysRevLett.110.173004

Pawlus, S., Paluch, M., and Dzida, M. (2010). Molecular dynamics changes induced by hydrostatic pressure in a supercooled primary alcohol. J. Phys. Chem. Lett. 1, 3249-3253. doi:10.1021/jz101288v

Petong, P., Pottel, R., and Kaatze, U. (1999). Dielectric relaxation of H-bonded liquids. Mixtures of ethanol and n-hexanol at different compositions and temperatures. J. Phys. Chem. A 103, 6114-6121. doi:10.1021/jp9910461

Power, G., Nagaraj, M., Vij, J. K., and Johari, G. P. (2011). Debye process and dielectric state of an alcohol in a nonpolar solvent. J. Chem. Phys. 134, 044525. doi:10.1063/1.3543713

Power, G., Vij, J. K., and Johari, G. P. (2007). Relaxations and nano-phase-separation in ultraviscous heptanol-alkyl halide mixture. J. Chem. Phys. 126, 034512. doi:10.1063/1.2409929

Sindzingre, P., and Klein, M. L. (1992). A molecular dynamics study of methanol near the liquid-glass transition. J. Phys. Chem. 96, 4681-4692. doi:10.1063/1.462805

Singh, L. P., and Richert, R. (2012). Watching hydrogen-bonded structures in an alcohol convert from rings to chains. Phys. Rev. Lett. 109, 167802. doi:10.1103/ PhysRevLett.109.167802

Souda, R. (2004). Glass transition and intermixing of amorphous water and methanol. Phys. Rev. Lett. 93, 235502. doi:10.1103/PhysRevLett.93.235502

Sugisaki, M., Suga, H., and Seki, S. (1968). Calorimetric study of the glassy state. III. Novel type calorimeter for study of glassy state and heat capacity of glassy methanol. Bull. Chem. Soc. Jpn 41, 2586-2591. doi:10.1246/bcsj.41.2586

Sun, M., Wang, L.-M., Tian, Y., Liu, R., Ngai, K. L., and Tan, C. (2011). Component dynamics in miscible mixtures of water and methanol. J. Phys. Chem. B 115, 8242-8248. doi:10.1021/jp202893v

Wang, L.-M., and Richert, R. (2004). Dynamics of glass-forming liquids. IX. Structural versus dielectric relaxation in monohydroxy alcohols. J. Chem. Phys. 121, 11170-11176. doi:10.1063/1.1811072

Wang, L.-M., and Richert, R. (2005). Debye type dielectric relaxation and the glass transition of alcohols. J. Phys. Chem. B 109, 11091-11094. doi:10.1021/jp051965d

Wang, L.-M., and Richert, R. (2005a). Identification of dielectric and structural relaxations in glass-forming secondary amides. J. Chem. Phys. 123, 054516. doi:10.1063/1.1997135

Wang, L.-M., and Richert, R. (2005b). Ideal mixing behavior of the Debye process in supercooled monohydroxy alcohols. J. Phys. Chem. B 109, 8767-8773. doi:10.1021/ jp050342x

Wang, L.-M., and Richert, R. (2007). Primary and secondary relaxation time dispersions in fragile supercooled liquids. Phys. Rev. B 76, 064201. doi:10.1103/ PhysRevB.76.064201

Wang, L.-M., Shahriari, S., and Richert, R. (2005). Diluent effects on the Debye-type dielectric relaxation in viscous monohydroxy alcohols. J. Phys. Chem. B 109, 23255-23262. doi:10.1021/jp054542k

Wang, L.-M., Tian, Y., Liu, R., and Richert, R. (2008). Calorimetric versus kinetic glass transitions in viscous monohydroxy alcohols. J. Chem. Phys. 128, 084503. doi:10.1063/1.2840357

Wang, L.-M., Velikov, V., and Angell, C. A. (2002). Direct determination of kinetic fragility indices of glassforming liquids by differential scanning calorimetry: kinetic versus thermodynamic fragilities. J. Chem. Phys. 117, 10184-10192. doi:10.1063/1.1517607

Zorębski, E., Dzida, M., and Wysocka, E. (2011). Acoustic and thermodynamic properties of 2-ethyl-1-hexanol by means of high-pressure speed of sound measurements at temperatures from (293 to 318) $\mathrm{K}$ and pressures up to $101 \mathrm{MPa}$. J. Chem. Eng. Data 56, 2680-2686. doi:10.1021/je100927m

Conflict of Interest Statement: The authors declare that the research was conducted in the absence of any commercial or financial relationships that could be construed as a potential conflict of interest.

Copyright $@ 2015$ Li, Chen, Gao, Tu and Wang. This is an open-access article distributed under the terms of the Creative Commons Attribution License (CC BY). The use, distribution or reproduction in other forums is permitted, provided the original author(s) or licensor are credited and that the original publication in this journal is cited, in accordance with accepted academic practice. No use, distribution or reproduction is permitted which does not comply with these terms. 